\section{ORIGINAL RESEARCH}

\author{
K. Dolic \\ K. Marr \\ V. Valnarov \\ M.G. Dwyer \\ E. Carl \\ Y. Karmon \\ C. Kennedy \\ C. Brooks \\ C. Kilanowski \\ K. Hunt \\ A.H. Siddiqui \\ D. Hojnacki
}

B. Weinstock-Guttman

R. Zivadinov

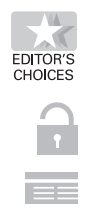

\title{
Intra- and Extraluminal Structural and Functional Venous Anomalies in Multiple Sclerosis, as Evidenced by 2 Noninvasive Imaging Techniques
}

BACKGROUND AND PURPOSE: Chronic cerebrospinal venous insufficiency (CCSVI) is a vascular condition characterized by anomalies of the main extracranial cerebrospinal venous routes that interfere with normal venous outflow. Research into CCSVI will determine its sensitivity and specificity for a diagnosis of MS, its prevalence in MS patients, and its clinical, MRI, and genetic correlates. Our aim was to investigate the prevalence and number of intra- and extraluminal structural and functional extracranial venous abnormalities by using DS and MRV, in patients with MS and HCs.

MATERIALS AND METHODS: One hundred fifty patients with MS, 104 (69.3\%) with RR and 46 (30.7\%) with a progressive MS course, and 63 age- and sex-matched HCs were scanned with 3T MR imaging by using TOF and TRICKS sequences (only patients with MS). All subjects underwent DS examination for intra- and extraluminal structural and functional abnormalities of the IJVs. Absent/pinpoint IJV flow morphology on MRV was considered an abnormal finding. Prominence of collateral extracranial veins was assessed with MRV.

RESULTS: Patients with MS had a significantly higher number of functional $(P<.0001)$, total $(P=$ $.001)$, and intraluminal $(P=.005)$ structural IJV DS abnormalities than HCs. There was a trend for more patients with MS with extraluminal IJV DS abnormalities $(P=.023)$. No significant differences were found on the MRV IJV flow morphology scale between patients with MS and HCs. Patients with progressive MS showed more extraluminal IJV DS abnormalities $(P=.01)$ and more MRV flow abnormalities on TOF $(P=.006)$ and TRICKS $(P=.01)$ than patients with nonprogressive MS. There was a trend for a higher number of collateral veins in patients with MS than in $\mathrm{HCs}(P=.016)$.

CONCLUSIONS: DS is more sensitive than MRV in detecting intraluminal structural and functional venous abnormalities in patients with MS compared with $\mathrm{HCs}$, whereas MRV is more sensitive in showing collaterals.

ABBREVIATIONS: CCSVI = chronic cerebrospinal venous insufficiency; $\mathrm{CV}=$ catheter venography; DS = Doppler sonography; EDSS = Expanded Disability Status Scale; Gd = gadolinium; HC = healthy control; ICC = interclass correlation coefficient; IJV = internal jugular vein; MRV = MR venography; $\mathrm{PP}=$ primary-progressive; $\mathrm{RR}=$ relapsing-remitting; $\mathrm{SP}=$ secondary-progressive; TOF = time-of-flight; TRICKS = time-resolved imaging of contrast kinetics; $V$ V = vertebral veins

M $\mathrm{S}$ is an autoimmune disease of the CNS that is a result of interaction between genetic, environmental, and infectious factors. ${ }^{1-4}$ Recently, a condition called CCSVI was found with high frequency in patients with MS. ${ }^{5}$ CCSVI presupposes that MS is associated with impaired venous drainage from the brain to the periphery due to venous anomalies in major extracranial neck and azygos veins. ${ }^{5}$

Recent studies by using extra- and transcranial DS, ${ }^{6-10}$

Received June 27, 2011; accepted after revision July 25.

From the Buffalo Neuroimaging Analysis Center (K.D., K.M., V.V., M.G.D., E.C., C.K., C.B., C.K., K.H., R.Z.), State University of New York, Buffalo, New York; and The Jacobs Neurological Institute (Y.K., D.H., B.W.-G, R.Z.), Department of Neurology, and Department of Neurosurgery (Y.K., A.H.S.), School of Medicine and Biomedical Sciences, Millard Fillmore Gates Hospital, Kaleida Health, University at Buffalo, State University of New York, Buffalo, New York.

Please address correspondence to Robert Zivadinov, MD, PhD, FAAN, Department of Neurology, School of Medicine and Biomedical Sciences, Buffalo Neuroimaging Analysis Center, 100 High St, Buffalo, NY 14203; e-mail: zivadinov@hotmail.com

Indicates open access to non-subscribers at www.ajnr.org

Indicates article with supplemental on-line tables.

http://dx.doi.org/10.3174/ajnr.A2877 phase-contrast and contrast-enhanced MRV, ${ }^{11-13}$ or $\mathrm{CV}^{14,15}$ failed to confirm the high frequency of abnormal cerebral venous outflow in patients with MS versus HCs, as originally reported. ${ }^{5}$

Previous CV studies in $\mathrm{MS}^{5,14-16}$ proposed that the extracranial venous anomalies are likely to be truncular venous malformations characterized by intraluminal defects (such as flaps, webs, septa, membranes, and malformed valves) or by extraluminal abnormalities represented by stenoses of the venous wall.

Therefore, in this study, we aimed to define the frequency of those venous abnormalities in patients with MS and HCs by using 2 noninvasive imaging techniques (DS and MRV).

\section{Materials and Methods}

\section{Subjects and Clinical Assessments}

The case-control prospective study included 150 consecutive patients with MS and 63 age- and sex-matched HCs who participated in the combined transcranial and extracranial venous Doppler study and fulfilled inclusion and exclusion criteria. ${ }^{6}$ The study started in April 2009 and is still enrolling subjects. Inclusion criteria for patients with 

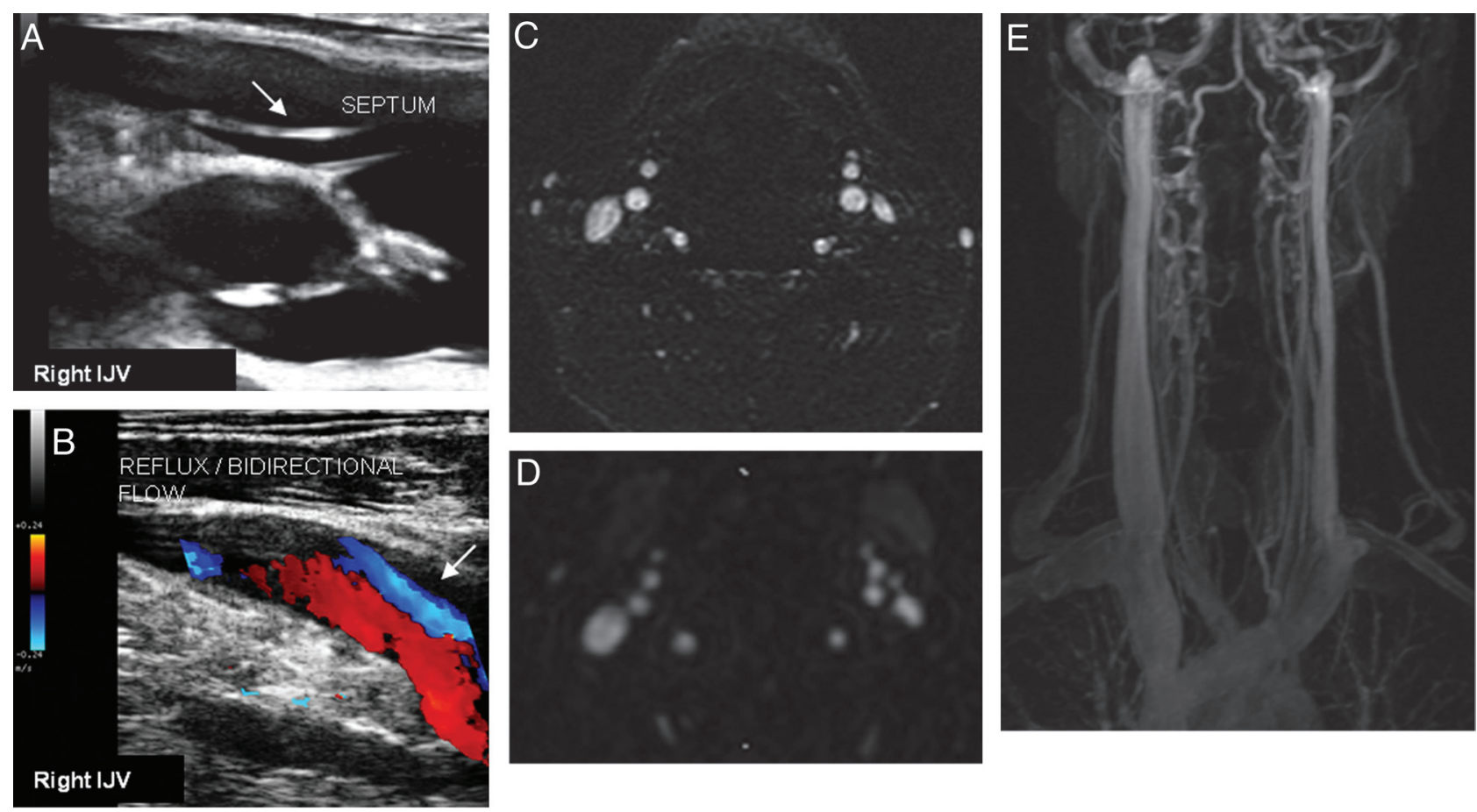

Fig 1. Intraluminal (septum, $A$ ) structural abnormality in the right IJV causing significant reflux/bidirectional flow directed toward the brain for a duration of $>0.88$ seconds in both the supine and sitting positions $(B)$, as detected by using DS, in a 33-year-old woman with RRMS with 2 years of disease duration and minimal disability (EDSS score, 1.0). 2D TOF (C) and enhanced 3D TRICKS ( $D$ and $E$ ) sequences show normal flow morphology.

MS were clinically definite MS, ${ }^{17}$ RR-, SP-, and PPMS disease course $^{18} ; 18-65$ years of age; an EDSS score from 0 to $6.5^{19}$; and diagnostic evaluation for extracranial venous anomalies by using DS and MRV examinations. Exclusion criteria were occurrence of relapse and steroid treatment in the 30 days preceding study entry, pre-existing medical conditions known to be associated with neck pathology, history of cerebral congenital vascular malformations, pregnancy, contraindication for receiving Gd-based contrast agents in patients with MS, history of chronic obstructive pulmonary disease, and arthritic necks (may not be able to lie flat). ${ }^{6}$ The study was approved by the local institutional review board, and informed consent was obtained from all subjects.

\section{DS}

Participants underwent extracranial DS of the neck. A color-coded DS scanner (MyLab 25; Esaote-Biosound, Irvine, California) equipped with a 7.5- to 10-Mhz transducer was used to examine venous return in the IJVs and VVs. All subjects were examined first in the supine and then in the sitting position $\left(0^{\circ}\right.$ and $\left.90^{\circ}\right)$. The DS examination was performed by 2 trained technologists who were blinded to subjects' characteristics, as previously described. ${ }^{6}$

The IJV abnormalities were divided into 3 subcategories (Figs 1-3): intraluminal structural (web, flap, septa, membrane, and malformed valve), extraluminal structural (stenosis and annulus), and functional abnormalities (presence of reflux/bidirectional flow, paradox, and no flow). The proposed classification and description of these abnormalities are given in Table 1.

Absence of detectable flow in VVs was considered abnormal and was compared with abnormal findings of VV on MRV.

To test the intra- and inter-rater reproducibility of the intra-, extraluminal, and functional venous abnormalities, 32 subjects $(20$ patients with MS, $7 \mathrm{HCs}$, and 5 controls with other neurologic dis- eases) were examined by 2 Doppler technicians who assessed all subjects twice during a 1-week period in a blinded manner. ${ }^{6}$

All DS examinations were double-checked and confirmed by 2 independent neuroradiologists in a blinded manner (D.H. and K.D.).

\section{MRV}

All patients with MS and HCs underwent unenhanced 2D-TOF and enhanced 3D-TRICKS sequences (in patients with MS) on 3T MR imaging (Figs 1 and 3). The participation of the HCs in the contrast portion of the MRV protocol was not recommended by our institutional review board.

\section{MRV Acquisition Protocol}

All subjects were examined on a 3T Signa Excite HD 12.0 TwinSpeed 8-channel scanner (GE Healthcare, Milwaukee, Wisconsin), with a maximum slew rate of $150 \mathrm{~T} / \mathrm{m} / \mathrm{s}$ and a maximum gradient amplitude in each orthogonal plane of $50 \mathrm{mT} / \mathrm{m}$ (zoom mode). A multichannel head and neck coil manufactured by GE Healthcare was used to acquire the following sequences: TOF and TRICKS MRV. The parameters used for TOF were the following: TR/TE, 17/4.3 ms; flip angle, $70^{\circ}$; 1.5 -mm section thickness; acquisition matrix, 320/192; and acquisition in the axial scan plane. The parameters used for TRICKS were the following: TR/TE, $4.2 / 1.6 \mathrm{~ms}$; flip angle, $30^{\circ}$; 2-mm section thickness; acquisition matrix, 320/192; and acquisition in the coronal scan plane. Intravenous Gd contrast was injected at a rate of $2 \mathrm{~mL} / \mathrm{s}$ by using a pressure injector. The total volume of contrast was $20 \mathrm{~mL}$. The scan protocol consisted of 18 phases of acquisition, each of 5 seconds' duration. The MR images were obtained from the level of the confluens sinuum to the level of the aortic arch.

The MRV analysis protocol has been previously described in detail. ${ }^{13}$ Briefly, the flow morphology of the IJVs was assessed on axial source TOF 

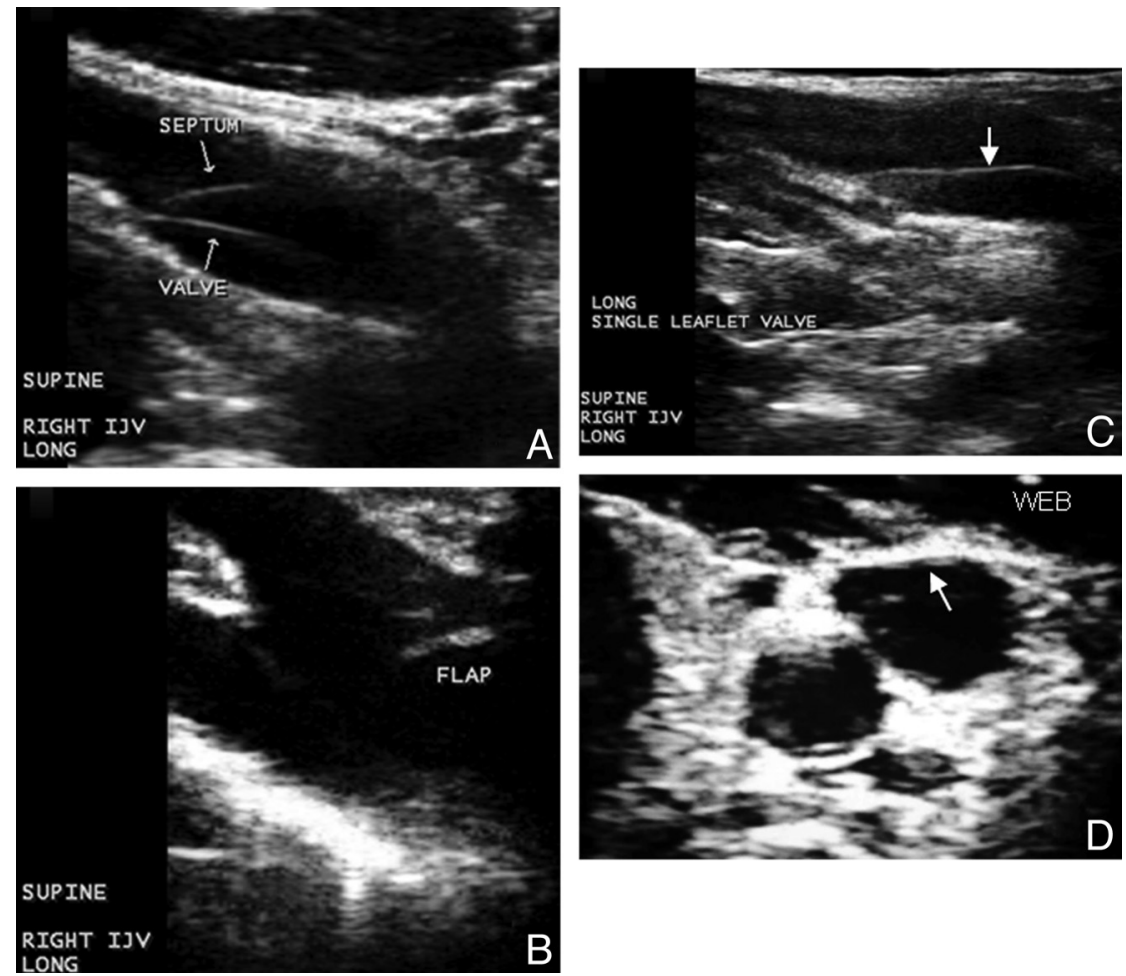

Fig 2. Examples of intraluminal structural abnormalities in the IJVs causing hemodynamic abnormality in HCs and patients with MS, 22 and 47 years of age. $A$, septum. $B$, Flap. $C$, Single leaflet valve. $D$, Web.
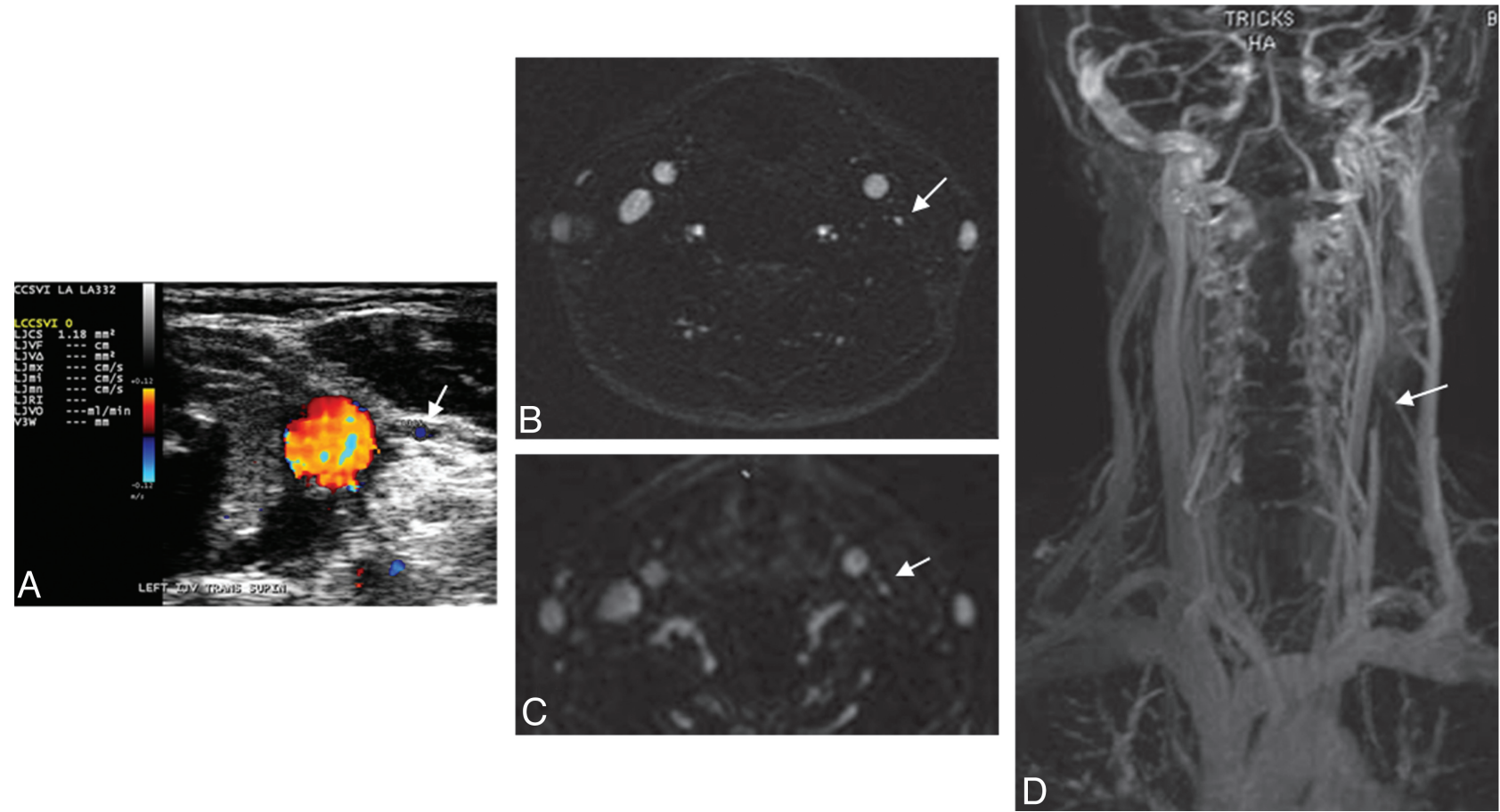

Fig 3. Extraluminal structural abnormality in the left IJV causing significantly reduced flow in both the supine and sitting positions, as detected by using DS $(A)$ and MRV (B-D) in a 46-year-old woman with SPMS with 20 years of disease duration and advanced disability (EDSS score, 5.0). 2D TOF (B) and enhanced 3D TRICKS (C and D) sequences show pinpoint flow morphology.

images as well as on axial reconstructed TRICKS sections. The flow was evaluated on an ordinal scale ranging from absent (no visible flow) to ellipsoidal (patent lumen). We assigned 5 qualitative flow-morphology categories: absent, pinpoint, flattened, crescentic, and ellipsoidal. ${ }^{13} \mathrm{We}$ considered only absent and pinpoint flow in the IJVs as abnormal. VV flow was classified as absent (abnormal)/present (normal).
We also assessed the left and right prominence of the other most important veins in the neck visible on MRV: external jugular veins, anterior jugular veins, facial veins, thyroid veins, and deep cervical veins, as previously reported. ${ }^{13}$ The prominence was graded as absent/present, and the number of collateral veins for the right and left side of the neck was also counted. 


\begin{tabular}{|c|c|}
\hline DS Classification & Types/Examples \\
\hline $\begin{array}{l}\text { Intraluminal structural venous abnormality; this is an echogenic structure } \\
\text { extending from the endothelial lining of the vein wall with/without } \\
\text { presence of functional abnormality; these abnormalities include web, } \\
\text { flap, septum, membrane, and malformed valve }\end{array}$ & $\begin{array}{l}\text { Web: multiple septa and/or flaps located in a cluster } \\
\text { Flap: thin linear echogenic structure extending from endothelial lining of vein } \\
\text { wall } \\
\text { Septum: thin linear echogenic structure extending from endothelial lining of vein } \\
\text { wall, and attached to it at both ends; septum may extend across a vein to } \\
\text { attach on opposing sides or attach on same side } \\
\text { Membrane: membranous structure almost occluding the entire diameter of the } \\
\text { vein } \\
\text { Malformed valve: dysdynamic or fibrous valve }\end{array}$ \\
\hline $\begin{array}{l}\text { Extraluminal structural venous abnormality; this is a restriction of the } \\
\text { venous wall or stenosis; these abnormalities include stenosis and } \\
\text { annulus }\end{array}$ & $\begin{array}{l}\text { Stenosis: CSA measurement of } \leq 3 \mathrm{~mm}^{2} \\
\text { Annulus: circumferential thickened vein wall that is restricting the vein from } \\
\text { fully expanding with respiratory or positional changes }\end{array}$ \\
\hline $\begin{array}{l}\text { Functional venous abnormality; this is an abnormal cerebral venous } \\
\text { outflow in the presence of a structural venous anomaly; these } \\
\text { abnormalities include reflux/bidirectional flow, paradox, and no flow }\end{array}$ & $\begin{array}{l}\text { Reflux/bidirectional flow: present in the IJV for }>0.88 \text { seconds with the head } \\
\text { at } 90^{\circ} \text { and } 0^{\circ} \\
\text { Paradox: vein wall not reacting to respiratory phase; noncompliant } \\
\text { No flow: no color flow noted in vein, despite deep breaths }\end{array}$ \\
\hline
\end{tabular}

Note:-CSA indicates cross-sectional area.

\begin{tabular}{|c|c|c|c|c|c|c|}
\hline & $\begin{array}{c}\text { MS } \\
(n=150)\end{array}$ & $\begin{array}{c}\mathrm{HC} \\
(n=63)\end{array}$ & $P$ & $\begin{array}{l}\text { NPRMS } \\
(n=104)\end{array}$ & $\begin{array}{c}\text { PRMS } \\
(n=46)\end{array}$ & $P$ \\
\hline Female sex (No.) (\%) & $102(68)$ & $36(57.1)$ & NS & $73(70.2)$ & $29(63)$ & NS \\
\hline Age (yr) (mean) (SD) & $43.2(10.6)$ & $42(15)$ & NS & $40.7(10.7)$ & 48.6 (8.2) & $<.001$ \\
\hline Age at onset, mean (SD) & $31.3(10)$ & - & - & $31.9(9.9)$ & $30(10.3)$ & NS \\
\hline Disease duration (mean) (SD) & $12.1(9.1)$ & - & - & $9.2(7)$ & $18.6(9.9)$ & $<.001$ \\
\hline Disease course (№.) (\%) & & - & & & & \\
\hline RR & $104(70.4)$ & & & $104(100)$ & & \\
\hline $\mathrm{SP}$ & $38(25.3)$ & & & & $38(82.6)$ & \\
\hline PP & $8(5.3)$ & & & & $8(17.4)$ & \\
\hline EDSS (mean) (SD) (median) & $3.4(9.1) 2.5$ & - & - & $2.5(1.4) 2.0$ & $5.8(1.7) 6.0$ & $<.001$ \\
\hline
\end{tabular}

Note:-NS indicates nonsignificant; NPR, nonprogressive; PR, progressive; -, not available.

a The differences between the study groups were tested using the $\chi^{2}$ test, Student's $t$ test, and Mann-Whitney U test.

All MRV scans were examined by 2 independent neuroradiologists in a blinded manner (D.H. and K.D.). The reproducibility results were previously reported. ${ }^{13}$

\section{Statistical Analyses}

Statistical analysis was performed by using the Statistical Package for the Social Sciences (Version 16.0; SPS, Chicago, Illinois). For descriptive statistics and estimates of prevalence and number of venous abnormalities, $t$ tests, Fisher exact tests, and the Mann-Whitney $U$ tests were used. Reproducibility was calculated by using the Cohen $\kappa$ and ICC tests. The relationship between different DS and MRV venous characteristics was assessed by using the Spearman rank correlation. Patients with MS were further divided into 2 groups: MS nonprogressive (RR) and MS progressive (SP and PP).

To correct for multiple comparisons, the nominal $P$ value $<.01$ was considered as significant by using 2-tailed tests.

\section{Results}

\section{Demographic and Clinical Characteristics}

Table 2 shows demographic and clinical characteristics of patients with MS and HCs. As expected, patients with progressive MS had significantly higher age and EDSS scores and longer disease duration than patients with nonprogressive MS (all $P<.001$ ). Of the 150 patients with MS, 95 (63.3\%) were on disease-modifying therapy.

\section{Reproducibility Results}

On-line Tables 1 and 2 show the intra- and inter-rater reproducibility for DS parameters. There was modest-to-high intrarater agreement for depicting total intra- and extraluminal structural and functional venous abnormalities. However, there was low-to-modest inter-rater agreement for the assessment of intraluminal and functional venous abnormalities and modest-to-high inter-rater agreement for the assessment of the extraluminal abnormalities.

\section{Intraluminal and Extraluminal Structural and Functional Venous Abnormalities in IJVs, as Evidenced by Doppler Sonography}

Table 3 shows the individual frequency and number of intraand extraluminal structural and functional IJV abnormalities, as evidenced by DS. Significantly more patients with MS presented with total $(P=.004)$ and intraluminal $(P=.008)$ structural and functional $(P=.003)$ abnormalities, with a trend toward more extraluminal abnormalities compared with $\mathrm{HCs}$ $(P=.044)$. There was a significantly higher number of total $(P=.001)$ and intraluminal structural $(P=.005)$ and functional abnormalities $(P<.0001)$, with a trend for a higher number of extraluminal structural $(P=.023)$ abnormalities in patients with MS compared with HCs. In patients with MS, there was a significant relationship between the higher number of intraluminal and functional abnormalities $(r=0.36$, 


\begin{tabular}{|c|c|c|c|c|c|c|}
\hline Individual Frequency of Venous Abnormalities & $\begin{array}{c}\mathrm{HC} \\
(n=63)\end{array}$ & $\begin{array}{c}\text { MS } \\
(n=150)\end{array}$ & $P$ Value $^{\mathrm{b}}$ & $\begin{array}{c}\text { NPRMS } \\
(n=104)\end{array}$ & $\begin{array}{c}\text { PRMS } \\
(n=46)\end{array}$ & $P$ Value $^{\mathrm{c}}$ \\
\hline Total structural (No.) (\%) & $34(54)$ & $111(74)$ & .004 & $72(69.2)$ & $39(84.8)$ & .033 \\
\hline Intraluminal structural (№.) (\%) & $31(49.2)$ & $102(68)$ & .008 & $67(64.4)$ & $35(76.1)$ & .110 \\
\hline Web & 0 & $6(4)$ & & $5(4.8)$ & $1(2.2)$ & \\
\hline Flap & $17(27)$ & $51(34)$ & & $36(34.6)$ & $15(32.6)$ & \\
\hline Septum & $9(14.3)$ & $43(28.7)$ & & $34(32.7)$ & $9(19.6)$ & \\
\hline Membrane & 0 & 0 & & 0 & 0 & \\
\hline Malformed valve & $18(28.6)$ & $40(26.7)$ & & $25(24)$ & $15(32.6)$ & \\
\hline Extraluminal structural (No.) (\%) & $7(11.1)$ & $33(22)$ & .044 & $17(16.3)$ & 16 (34.8) & .01 \\
\hline Stenosis & $7(11.1)$ & $33(22)$ & & $17(16.3)$ & $16(34.8)$ & \\
\hline Annulus & 0 & 0 & & 0 & 0 & \\
\hline Total functional (No.) (\%) & $21(33.3)$ & $82(54.7)$ & .003 & 55 (52.9) & $27(58.7)$ & .316 \\
\hline Reflux & $20(31.7)$ & $74(49.3)$ & & $49(47.1)$ & $25(54.3)$ & \\
\hline Paradox & 0 & $2(1.3)$ & & $1(1)$ & $1(2.2)$ & \\
\hline No flow & $2(3.2)$ & $16(10.7)$ & & $8(7.7)$ & $8(17.4)$ & \\
\hline \multicolumn{7}{|l|}{ No. of venous abnormalities } \\
\hline Total structural (mean) (SD) & $1.05(1.3)$ & $1.85(1.8)$ & .001 & $1.75(1.7)$ & $2.04(1)$ & .384 \\
\hline Intraluminal structural (mean) (SD) & $0.9(1.2)$ & $1.53(1.7)$ & .005 & $1.5(1.6)$ & $1.61(1.9)$ & .805 \\
\hline Web & 0 & $0.07(0.4)$ & & $0.07(0.3)$ & $0.09(0.6)$ & \\
\hline Flap & $0.33(0.6)$ & $0.56(1)$ & & $0.56(1)$ & $0.57(1)$ & \\
\hline Septum & $0.24(0.7)$ & $0.55(1)$ & & $0.57(1)$ & $0.5(1.2)$ & \\
\hline Membrane & 0 & 0 & & 0 & 0 & \\
\hline Malformed valve & $0.35(0.6)$ & $0.37(0.7)$ & & $0.33(0.6)$ & $0.5(0.8)$ & \\
\hline Extraluminal structural, (mean) (SD) & $0.13(0.5)$ & $0.31(0.7)$ & .023 & $0.25(0.7)$ & $0.43(0.7)$ & .01 \\
\hline Stenosis & $0.13(.5)$ & $0.31(0.7)$ & & $0.25(0.7)$ & $0.43(0.7)$ & \\
\hline Annulus & 0 & 0 & & 0 & 0 & \\
\hline Total functional (mean) (SD) & $0.51(0.9)$ & $1.13(1.2)$ & $<.0001$ & $1.02(1.2)$ & $1.4(1.3)$ & .096 \\
\hline Reflux & $0.44(0.8)$ & $0.88(1.1)$ & & $0.85(1.1)$ & $0.96(1.2)$ & \\
\hline Paradox & 0 & $0.02(0.2)$ & & $0.02(0.2)$ & $0.02(0.1)$ & \\
\hline No flow & $0.06(0.6)$ & $0.17(0.5)$ & & $0.13(0.5)$ & $0.26(0.6)$ & \\
\hline
\end{tabular}

Note:-NPR indicates nonprogressive; PR, progressive.

a The frequency differences of the structural and functional venous abnormalities between the study groups were tested using the Fisher exact test, whereas the number of venous abnormalities differences was tested using Mann-Whitney $\mathrm{U}$ test.

${ }^{b} P$ value represents comparison between $\mathrm{HC}$ and $\mathrm{MS}$.

c $P$ value represents comparison between patients with NPR- and PRMS.

Table 4: Flow morphology of IJVs in patients with MS and HCs 2D-TOF using venography and 3D-TRICKS

\begin{tabular}{|c|c|c|c|c|c|c|}
\hline \multirow[b]{2}{*}{ Morphology Score } & \multicolumn{4}{|c|}{ TOF } & \multicolumn{2}{|c|}{ TRICKS } \\
\hline & $\begin{array}{c}\mathrm{HC} \\
(n=63)\end{array}$ & $\begin{array}{c}\text { MS } \\
(n=150)\end{array}$ & $\begin{array}{c}\text { NPRMS } \\
(n=104)\end{array}$ & $\begin{array}{c}\text { PRMS } \\
(n=46)\end{array}$ & $\begin{array}{c}\text { NPRMS } \\
(n=96)\end{array}$ & $\begin{array}{c}\text { PRMS } \\
(n=44)\end{array}$ \\
\hline Absent (No.) (\%) & $5(7.9)$ & $23(15.3)$ & $14(13.5)$ & $9(19.6)$ & $7(6.7)$ & $2(4.3)$ \\
\hline Pinpoint (No.) (\%) & $12(19)$ & $23(15.3)$ & $12(11.5)$ & $11(23.9)$ & $18(17.3)$ & $17(37)$ \\
\hline Flattened (No.) (\%) & $18(28.6)$ & $50(33.3)$ & $33(31.7)$ & $17(37)$ & $43(41.3)$ & $30(65.2)$ \\
\hline Crescentic (No.) (\%) & $2(3.2)$ & $2(1.3)$ & $2(1.9)$ & $0(0)$ & $3(2.9)$ & $1(2.2)$ \\
\hline Ellipsoidal (No.) (\%) & $26(41.3)$ & $52(34.7)$ & $43(41.3)$ & $9(19.6)$ & 93 (89.4) & 41 (89.1) \\
\hline$P$ value & \multicolumn{2}{|c|}{.259} & \multicolumn{2}{|c|}{.006} & \multicolumn{2}{|c|}{.01} \\
\hline
\end{tabular}

Note:-NPR indicates nonprogressive; PR, progressive.

a The differences on the ordinal morphologic flow scale between the study groups were evaluated using the Mann-Whitney U test. Only absent and pinpoint flows in the IJVs were considered abnormal.

$P=<.0001)$, extraluminal and functional abnormalities $(r=$ $0.29, P=.008)$, and intraluminal and extraluminal abnormalities $(r=0.23, P=.006)$.

There were significantly more patients with extraluminal structural abnormalities $(P=.01)$ and a higher number of extraluminal abnormalities $(P=.01)$ in patients with progressive MS compared with those with nonprogressive MS. There was also a trend toward more patients with total structural abnormalities $(P=.033)$. No significant differences were observed between patients with progressive and nonprogressive MS for intraluminal structural and functional abnormalities.

No significant relationship was found in patients with MS or HCs between age and sex, and the frequency and number of structural and functional IJV abnormalities, except a trend toward greater age and a higher number of functional IJV abnormalities in patients with progressive MS $(r=0.33$, $P=.026)$.

\section{MRV Findings in IJVs}

Table 4 shows MRV findings in patients with MS and HCs on TOF and in progressive and nonprogressive MS subgroups on TOF and TRICKS MR imaging sequences. No significant differences were found on the flow-morphologic scale between patients with MS and HCs. Abnormal TOF findings (absent/ pinpoint flow morphology) were found in $46(30.7 \%)$ of the patients with MS and 17 (27\%) HCs.

Significantly more abnormalities were observed on the flow-morphologic scale in patients with progressive compared 
with nonprogressive MS on TOF $(P=.006)$ and TRICKS $(P=.01)$. In total, $20(43.5 \%)$ patients with progressive MS had abnormal findings on TOF, and 19 (43.2\%), on TRICKS. The figures were $26(25 \%)$ in patients with nonprogressive MS for TOF and 25 (26\%) for TRICKS. The relationship between TOF and TRICKS findings was highly related $(r=0.95$, $P<.001)$.

\section{Collateral Vein Findings}

There was a trend toward a higher mean number of collateral veins in patients with MS compared with HCs on TOF (2.56 versus $2.1, P=.016)$. No significant differences were observed in the number of collateral veins between patients with progressive and nonprogressive MS on TOF (2.65 versus 2.52, $P=.715)$ or on TRICKS (2.57 versus $2.62, P=.756$ ).

Patients with MS with a higher number of functional and intraluminal IJV DS abnormalities showed a significantly higher number of collateral veins on TRICKS $(r=0.27$, $P=.009$ and $r=0.23, P=.01$, respectively) and on TOF ( $r=0.27, P=.006$ and $r=0.22, P=.01$, respectively).

\section{VV Findings}

There was absent flow in the VVs of 6 (9.5\%) HCs and of 16 $(10.7 \%)$ patients with MS on DS $(P=.932)$. No significant differences were observed between patients with progressive and nonprogressive MS in VV flow on DS and MRV.

\section{Discussion}

This study investigated the intra- and extraluminal structural and functional extracranial venous abnormalities and the potential value of MRV and DS in depicting those abnormalities. Patients with MS showed a significantly higher number of total and intraluminal structural and functional abnormalities on DS compared with HCs. No differences on the morphologic MRV flow scale were found between patients with MS and HCs. Patients with progressive MS presented with significantly more extraluminal DS abnormalities and more flow abnormalities on MRV than those with nonprogressive MS.

From the time it was first mentioned, the CCSVI theory has not ceased to provoke controversy and attention in the scientific community ${ }^{20-23}$ and the media. The originally reported sensitivity and specificity of the CCSVI criteria for MS diagnosis $^{5}$ were not reproduced by recent DS, ${ }^{6-10} \mathrm{MRV},{ }^{11-13}$ or $\mathrm{CV}^{14,15}$ studies. A major point that can explain different findings between the original and subsequent studies is that the CCSVI diagnosis is mainly based on extra- and transcranial echo-color DS criteria, which are operator-dependent and not easy to blind in a clinical setting. Moreover, the value of the CCSVI criteria is controversial because they combine functional and structural intra- and extracranial venous abnormalities in a single binary composite. ${ }^{24}$ The assessment of the second CCSVI criterion (reflux in deep cerebral veins) is particularly controversial because the direction of the blood flow in veins connecting cortical with deep veins may vary considerably as a consequence of the physiologic interindividual variation of the cerebral venous anatomy. ${ }^{6,7,9,24}$ In addition, the value of MRV-based techniques for a diagnosis of CCSVI has not yet been completely elucidated. ${ }^{13,25}$ To provide better evidence on the types of venous anomalies, we focused, in this study, on the assessment of extracranial neck veins by
2 different noninvasive imaging techniques. Furthermore, we divided venous abnormalities into structural (intra- and extraluminal) and functional, to better understand their contribution to the CCSVI concept.

The DS examination showed that a substantial number of patients with MS and HCs presented with at least 1 structural venous abnormality in their IJVs. Similar results were found for the number of intraluminal abnormalities in the IJVs. More patients with MS and HCs presented with intraluminal abnormalities compared with the extraluminal ones. Of all the intraluminal abnormalities examined, the septum and flap occurred most frequently in patients with MS as well as in HCs, while no difference between the groups was found for the malformed valve (impaired mobility or thickened fibrotic valve), in line with a recent report. ${ }^{9}$ No membrane abnormalities were found in patients with MS and HCs.

All in all, these results suggest that the most frequent venous abnormalities in the IJVs, indicative of CCSVI, are of intraluminal origin. Although head and neck veins are clearly shown by using MRV, this technique does not have the resolution to visualize intraluminal abnormalities. This is 1 of the main limitations when comparing MRV with DS, as discussed in a recent multimodal diagnostic study. ${ }^{25}$ The fact that intraluminal abnormalities present the most frequent type of venous abnormality in the CCSVI criteria ${ }^{5,6,10}$ can further explain the discrepant results between $\mathrm{DS}^{5,6}$ and MRV studies. ${ }^{11-13}$ Although our intrarater reproducibility results showed modest-to-high agreement for the detection of the type and number of intraluminal abnormalities, the inter-rater agreement for these abnormalities showed low reproducibility for the detection of type and modest reproducibility for the detection of number. In the present study, the presence of intraluminal abnormalities did not imply the presence of functional abnormality or significant IJV stenosis (cross-sectional area, $\leq 0.3 \mathrm{~cm}^{2}$ ). Therefore, the results from this study suggest that $\mathrm{HCs}$ also present frequently with intraluminal abnormalities, as recently reported. ${ }^{6,10}$ The possible origin of these abnormalities could be congenital, ${ }^{26}$ aging-dependent, or a possible consequence of an inflammatory process. ${ }^{6}$ Therefore, further research is needed to determine whether these anomalies represent a pathologic condition or a physiologic variation.

No significant intraluminal differences were seen in patients with progressive versus nonprogressive MS, which could indicate that intraluminal abnormalities may appear earlier in the disease process. However, we found that age in patients with progressive MS correlated with the presence of DS intraluminal abnormalities. Although we did not find a relationship between age and the number of intraluminal abnormalities either in HCs or in the entire MS group, it cannot be excluded that the prevalence of these abnormalities is aging-dependent. A recent study that investigated IJV changes with aging in HCs found a decreased proportion of venous drainage and increased IJV reflux prevalence in older subjects. ${ }^{27}$

In the present study, a trend toward a higher prevalence and number of extraluminal abnormalities was found in patients with MS compared with HCs on DS, whereas more patients with progressive-versus-nonprogressive MS presented with those anomalies. The presence of an annulus was not detected, contrary to previously reported results. ${ }^{5,16}$ Both 
intra- and inter-rater reproducibility showed modest-to-high agreement for the presence and number of extraluminal abnormalities. Therefore, our reproducibility data suggest that DS evaluation of extraluminal abnormalities is less operatordependent and could be more reliable in the evaluation of the venous anomalies in the IJVs.

On DS, patients with MS showed a significantly higher prevalence and number of functional abnormalities in the IJVs than HCs. No differences were seen between patients with progressive and nonprogressive MS. The most prevalent abnormality was reflux in patients with MS and in HCs, followed by no flow. A recent study found a strong correlation between jugular venous reflux and age-related white matter changes in elderly healthy subjects. ${ }^{28}$ Although a significant relationship was found between the number of functional and the number of intra- and extraluminal abnormalities, this relationship could explain, at best, only $20 \%$ of the variance. In addition, modest-to-high intrarater agreement for the detection of type and number of functional abnormalities was not observed for inter-rater agreement. More quantitative measures for the definition of functional abnormalities such as blood flow velocity and blood volume flow could be potentially more reliable in assessing the degree of venous outflow obstruction in the IJVs. ${ }^{7}$

An attempt was made to distinguish intra- versus extraluminal structural and functional abnormalities in the IJVs with both MRV techniques used in this study, but ultimately, this distinction was not possible. ${ }^{13,25}$ Consequently, all MRV abnormalities were classified as flow abnormalities due to possible intra- or extraluminal origin. No significant differences on the morphologic flow MRV scale were found between patients with MS and HCs, in line with 3 recently published smaller scale MRV venous flow studies. ${ }^{11-13}$ In a recent study, ${ }^{13}$ modest-to-low scan-rescan reproducibility agreement between baseline and follow-up MRV was detected. Nevertheless, we found significantly more flow abnormalities in patients with progressive versus nonprogressive MS both on TOF and TRICKS, confirming the DS extraluminal results. One of the limitations in the present study is the use of conventional MRV techniques, which are more prone to artifacts compared with other more advanced approaches. ${ }^{12,29} \mathrm{Al}-$ though we did not perform contrast MRV sequences in HCs as recommended by our institutional review board, we showed high correlation between TOF and TRICKS in the present and previous studies. $^{25,30}$

In the present study, there was a trend toward a higher number of collateral veins in patients with MS compared with HCs, contrary to our previous study. ${ }^{13}$ Patients with MS with a higher number of DS intraluminal IJV flow abnormalities showed a significantly higher number of collateral veins on both MRV sequences. No significant difference in the number of collaterals between patients with nonprogressive versus progressive MS was found. These results indeed provide an important finding, because they support the existence of collateral circulation as a possible compensatory mechanism for the higher number of functional and intraluminal abnormalities in the IJVs, as previously proposed. ${ }^{5,16}$ It could be hypothesized that collaterals develop as a compensatory mechanism for the presence of intraluminal structural flow abnormalities; and when their compensatory ability is overcome, extralumi- nal abnormalities begin to develop. This theory is supported by recent work of Yamout at al, ${ }^{14}$ who performed CV on 42 patients with MS in various phases of the disease (at onset and after 5 and 10 years) and found that extracranial venous stenosis was very rare at onset but became more frequent in subjects with longer MS duration. We considered the use of DS for visualizing collaterals; however, due to Doppler inability to follow the complete course of the collateral vein, we were unable to identify the vein with $100 \%$ accuracy. Therefore, MRV is more accurate than DS for following and labeling collaterals of the extracranial venous system. In a majority of cases, there was also a correlation between the size of the ipsilateral IJV and collateral veins, meaning that prominent collaterals followed stenotic IJVs with no visible flow. However, there were cases with normal patent IJV lumens and prominent collaterals. If the IJVs are developmentally smaller, then the other veins would likely be more prominent. Longitudinal studies should investigate the evolution of collaterals in stenotic or developmentally smaller IJVs.

We did not find VV flow differences on MRV and DS between patients with MS and HCs or between MS subgroups, which is in line with recent studies. ${ }^{13,25}$

The value of DS and MRV for screening of CCSVI was tested against $\mathrm{CV}$ with promising results in 2 recent pilot studies that included patients with MS and HCs. ${ }^{25,30}$ In 1 of these studies, DS showed $82 \%$ sensitivity, $100 \%$ specificity, and 99\% positive and 95\% negative predictive values compared with CV in 10 patients with MS. ${ }^{25}$ However, another recent study found no overlap between CV and DS in 7 of the 8 patients who presented with a sonographic finding of CCSVI. ${ }^{9}$ A multimodal approach by using noninvasive and invasive diagnostic techniques is recommended to determine the degree of extracranial venous structural and functional impairment in patients with MS and HCs.

One of the limits of this study is that we did not use invasive $\mathrm{CV}$ to confirm the DS and MRV findings. Although CV is considered the criterion standard for assessing and grading endovascular stenoses, $\mathrm{CV}$ is merely lumenography, providing little or no data on the wall of the vessel or its intraluminal structures. Malformed and/or reversed valve cusps can be crossed by the catheter and kept open artificially, thereby preventing documentation of stenosis. ${ }^{24}$ No established criteria or guidelines currently exist on $\mathrm{CV}$ for the detection of venous anomalies indicative of CCSVI. ${ }^{24}$ Any venous lumen reduction $>50 \%$ on CV is considered a sign of significant stenosis, which is somewhat arbitrary. ${ }^{5,16}$ More sophisticated CV categoric criteria (ranging from grade 1 to grade 4) were recently proposed. ${ }^{15}$ Our preliminary experience with $\mathrm{CV}^{25,30}$ indicates that proper training is needed for recognition of pathologic intraluminal anomalies indicative of CCSVI and that more sophisticated criteria for detection of these anomalies, such as time to empty contrast from veins or waisting of the balloon, need to be tested and validated. ${ }^{24}$ In addition, $\mathrm{CV}$ is an invasive method that requires radiation exposure, and there are ethical concerns in applying this technique to a large group of HCs.

Another limitation of our study is that we did not measure directly the venous pressures and flow patterns in the dural venous sinuses (the common pathway out of the brain before the cervical veins) to demonstrate any evidence of increased 
pressure or abnormal flow patterns, such as reflux or bidirectional flow. Additionally, because there are no indications that patients with sinus thrombosis and elevated intracranial pressures or those with jugular occlusions or resections (eg, with radical neck dissections) ever develop demyelinating lesions, ${ }^{20}$ our DS and MRV findings need further evaluation.

\section{Conclusions}

Despite the limitations in comparing different imaging techniques and the use of different imaging criteria, we found that conventional MRV has limited value for detection of extracranial venous anomalies compared with DS because it cannot distinguish intraluminal structural and functional venous abnormalities. However, MRV is more sensitive for showing collaterals.

\section{Acknowledgments}

We thank Eve Salczynski for technical assistance in the preparation of this manuscript.

Disclosures: Robert Zivadinov—Related: Grant. Direct MS Foundation,* Unrelated: Consultancy. Teva Neuroscience, Biogen Idec, Grants/Grants Pending. Teva Neuroscience, Biogen Idec, EMD Serono, Greatbatch, Payment for Lectures lincluding service on Speakers Bureaus): Teva Neuroscience, Biogen Idec. Adnan H. Siddiqui-Related: Grant. National Institutes of Health (coinvestigator: National Institute of Neurological Disorders and Stroke 1R01NS064592-01A1, hemodynamic induction of pathologic remodeling leading to intracranial aneurysms), University at Buffalo (Research Development Award), Consulting Fee or Honorarium: Codman \& Shurtleff Inc, Concentric Medical, ev3/Covidien Vascular Therapies, GuidePoint Global consulting, Penumbra, Abbott Vascular, courses for American Association of Neurological Surgeons, Genentech, Neocure Group LLC, an Emergency Medicine Conference, Abbott Vascular and Codman \& Shurtleff Inc for training other neurointerventionists in carotid stenting and for training physicians in endovascular stenting for aneurysms, University of lowa Endovascular Course, Unrelated: Board Membership: Codman \& Shurtleff, Comments: Advisory Board; Payment for Lectures (including service on Speakers Bureaus): Codman \& Shurtleff Inc, Genentech; Stock/Stock Options: Hotspur, Intratech Medical, StimSox, Valor Medical. Bianca Weinstock-Guttman-Unrelated: Consultancy: Biogen Idec, Teva Neuroscience, EMD Serono, Pfizer, Acorda, Grants/Grants Pending: Biogen Idec, ${ }^{*}$ EMD Serono, ${ }^{*}$ Acorda, ${ }^{*}$ Novartis, ${ }^{*}$ Shire, ${ }^{*}$ Teva Neuroscience, Payments for Lectures (including service on Speakers Bureaus): Biogen Idec, EMD Serono, Teva Neuroscience, Pfizer, Acorda, Novartis. *Money paid to the institution.

\section{References}

1. Compston A, Coles A. Multiple sclerosis. Lancet 2008;372:1502-17

2. Frohman EM, Racke MK, Raine CS. Multiple sclerosis: the plaque and its pathogenesis. N Engl J Med 2006;354:942-55

3. Weinshenker BG, Bass B, Rice GP, et al. The natural history of multiple sclerosis: a geographically based study. I. Clinical course and disability. Brain 1989;112(pt 1):133-46

4. Ascherio A, Munger KL. Environmental risk factors for multiple sclerosis. Part II. Noninfectious factors. Ann Neurol 2007;61:504-13

5. Zamboni $\mathrm{P}$, Galeotti R, Menegatti E, et al. Chronic cerebrospinal venous insufficiency in patients with multiple sclerosis. J Neurol Neurosurg Psychiatry 2009;80:392-99

6. Zivadinov R, Marr K, Cutter G, et al. Prevalence, sensitivity, and specificity of chronic cerebrospinal venous insufficiency in MS. Neurology 2011;77:138-44
7. Doepp F, Paul F, Valdueza JM, et al. No cerebrocervical venous congestion in patients with multiple sclerosis. Ann Neurol 2010;68:173-83

8. Krogias C, Schroder A, Wiendl H, et al. "Chronic cerebrospinal venous insufficiency" and multiple sclerosis: critical analysis and first observation in an unselected cohort of MS patients [in German]. Nervenarzt 2010;81:740-46

9. Baracchini C, Perini $P$, Calabrese $M$, et al. No evidence of chronic cerebrospinal venous insufficiency at multiple sclerosis onset. Ann Neurol 201 1;69:90-99

10. Mayer CA, Pfeilschifter W, Lorenz MW, et al. The perfect crime? CCSVI not leaving a trace in MS. J Neurol Neurosurg Psychiatry 2011;82:436-40

11. Sundstrom P, Wahlin A, Ambarki K, et al. Venous and cerebrospinal fluid flow in multiple sclerosis: a case-control study. Ann Neurol 2010;68:255-59

12. Wattjes MP, van Oosten BW, de Graaf WL, et al. No association of abnormal cranial venous drainage with multiple sclerosis: a magnetic resonance venography and flow-quantification study. J Neurol Neurosurg Psychiatry 2011; 82:429-35

13. Zivadinov R, Lopez-Soriano A, Weinstock-Guttman B, et al. Use of MR venography for characterization of the extracranial venous system in patients with multiple sclerosis and healthy control subjects. Radiology 2011;258:562-70

14. Yamout B, Herlopian A, Issa Z, et al. Extracranial venous stenosis is an unlikely cause of multiple sclerosis. Mult Scler 2010;16:1341-48

15. Ludyga T, Kazibudzki M, Simka M, et al. Endovascular treatment for chronic cerebrospinal venous insufficiency: is the procedure safe? Phlebology 2010;25:286-95

16. Zamboni P, Galeotti R, Menegatti E, et al. A prospective open-label study of endovascular treatment of chronic cerebrospinal venous insufficiency. $J$ Vasc Surg 2009;50:1348-58, e1-3

17. Polman CH, Reingold SC, Edan G, et al. Diagnostic criteria for multiple sclerosis: 2005 revisions to the "McDonald Criteria." Ann Neurol 2005; 58:840-46

18. Lublin FD, Reingold SC. Defining the clinical course of multiple sclerosis: results of an international survey-National Multiple Sclerosis Society (USA) Advisory Committee on Clinical Trials of New Agents in Multiple Sclerosis. Neurology 1996;46:907-11

19. Kurtzke JF. Rating neurologic impairment in multiple sclerosis: an Expanded Disability Status Scale (EDSS). Neurology 1983;33:1444-52

20. Khan O, Filippi M, Freedman MS, et al. Chronic cerebrospinal venous insufficiency and multiple sclerosis. Ann Neurol 2010;67:286-90

21. Rudick RA. Multiple sclerosis: is multiple sclerosis caused by venous insufficiency? Nat Rev Neurol 2010;6:472-74

22. Filippi M, Rocca MA, Barkhof F, et al. Multiple sclerosis and chronic cerebrospinal venous insufficiency: the neuroimaging perspective. AJNR Am J Neuroradiol 2011;32:424-47

23. D'Haeseleer M, Cambron M, Vanopdenbosch L, et al. Vascular aspects of multiple sclerosis. Lancet Neurol 2011;10:657-66

24. Zivadinov R, Ramanathan M, Dolic $\mathrm{K}$, et al. Chronic cerebrospinal venous insufficiency in multiple sclerosis: diagnostic, pathogenetic, clinical and treatment perspectives. Expert Rev Neurother 2011;11:1277-94

25. Zivadinov R, Galeotti R, Hojnacki D, et al. Value of MR venography for detection of internal jugular vein anomalies in multiple sclerosis: a pilot longitudinal study. AJNR Am I Neuroradiol 2011;32:938-46

26. Lee AB, Laredo J, Neville R. Embryological background of truncular venous malformation in the extracranial venous pathways as the cause of chronic cerebrospinal venous insufficiency. Int Angiol 2010;29:95-108

27. Chung C, Lin Y, Chao A, et al. Jugular venous hemodynamic changes with aging. Ultrasound Med Biol 2010;36:1776-82

28. Chung CP, Wang PN, Wu YH, et al. More severe white matter changes in the elderly with jugular venous reflux. Ann Neurol 2011;69:553-59

29. van Amerom JF, Vidarsson L, Wu S, et al. Regional pulmonary blood flow: comparison of dynamic contrast-enhanced MR perfusion and phase-contrast MR. Magn Reson Med 2009;61:1249-54

30. Hojnacki D, Zamboni P, Lopez-Soriano A, et al. Use of neck magnetic resonance venography, Doppler sonography and selective venography for diagnosis of chronic cerebrospinal venous insufficiency: a pilot study in multiple sclerosis patients and healthy controls. Int Angiol 2010;29:127-39 